\title{
Pancreatic cancer and adaptive metabolism in a nutrient-deficient environment
}

\author{
ROBERT SŁOTWIŃSKI ${ }^{1}$, SYLWIA MAŁGORZATA SŁOTWIŃSKA ${ }^{2}$
}

${ }^{1}$ Department of Immunology, Biochemistry and Nutrition, Medical University of Warsaw, Warsaw, Poland

${ }^{2}$ Department of Oral Hygiene, Medical University of Warsaw, Warsaw, Poland

\begin{abstract}
Despite tremendous progress in the treatment of many cancer types, leading to a significant increase in survival, pancreatic ductal adenocarcinoma (PDAC) is still burdened with high mortality rates (5-year survival rate < 9\%) due to late diagnosis, aggressiveness, and a lack of more effective treatment methods. Early diagnosis and new therapeutic approaches based on the adaptive metabolism of the tumor in a nutrient-deficient environment are expected to improve the future treatment of PDAC patients. It was found that blocking selected metabolic pathways related to the local adaptive metabolic activity of pancreatic cancer cells, improving nutrient acquisition and metabolic crosstalk within the microenvironment to sustain proliferation, may inhibit cancer development, increase cancer cell death, and increase sensitivity to other forms of treatment (e.g., chemotherapy). The present review highlights selected metabolic signaling pathways and their regulators aimed at inhibiting the neoplastic process. Particular attention is paid to the adaptive metabolism of pancreatic cancer, including fatty acids, autophagy, macropinocytosis, and deregulated cell-surface glycoproteins, which promotes cancer cell development in an oxygen-deficient and nutrient-poor environment.
\end{abstract}

Key words: pancreatic carcinoma, adaptive metabolism, autophagy, macropinocytosis, mucins.

(Cent Eur J Immunol 2021; 46 (3): 388-394)

\section{Introduction}

Pancreatic cancer remains the most aggressive cancer and due to late diagnosis the 5-year survival rate still stands at just 9\% [1]. Despite recent improvements in the systemic therapy of pancreatic cancer (pancreatic ductal adenocarcinoma - PDAC), initially with demonstration of the activity of the FOLFIRINOX regimen, and subsequently with the approval of nab-paclitaxel in combination with gemcitabine (GEM), the prognosis still remains poor [2]. According to the APACT trial (updated version 6 January 2020), there was no significant benefit in the median disease-free survival for gemcitabine plus nab-paclitaxel vs. gemcitabine alone in the adjuvant setting of PDAC treatment [3]. Due to the high mortality and limited effectiveness of standard treatments, resulting from the high aggressiveness of PDAC, increasing attention has been paid to the basic molecular mechanisms that enable local tumor development and metastasis formation. Of particular interest, both in diagnostic and therapeutic terms, are the disturbances in signaling pathways associated with important energy sources for pancreatic cancer cells. An important diagnostic element has emerged from metabolic disorders related to the local adaptive metabolism of pancreatic cancer, which affect the energy and nutritional requirements and cancer aggressiveness, including proliferation rate, metastasis formation, resistance to apoptosis and chemotherapy [4]. Despite the large number of studies, mainly experimental, on pancreatic cancer cell lines, there is still no answer to the basic question of whether the assessment of metabolic reprogramming will be helpful in the early diagnosis of pancreatic cancer and the initiation of more effective therapy.

A better understanding of metabolic signaling pathways' reprogramming and the possibilities of their early regulation is at the center of interest of many scientists who hope to inhibit pancreatic cancer progression. The metabolic mediators of glucose, glutamine, lipids, nucleotides, amino acids, and mitochondrial metabolism have emerged as novel therapeutic targets. The roles of autophagy, macropinocytosis, lysosomal transport, recycling, amino acid transport, lipid transport, and reactive oxygen species in pancreatic cancer development are also being extensively studied [4]. One of the main goals of these studies is to identify tumor "checkpoints" for the mechanisms that drive or inhibit cell proliferation and metastasis formation in order to determine their accuracy for individualization of the therapeutic approach. The present review highlights selected metabolic signaling pathways and

Correspondence: Prof. Sylwia Małgorzata Słotwińska, Department of Oral Hygiene, Medical University of Warsaw, Warsaw, Poland, e-mail: sylwia.slotwinska@wum.edu.pl

Submitted: 21.04.2021; Accepted: 5.05.2021 
their regulators aimed at inhibiting the neoplastic process. Particular attention is paid to the adaptive metabolism of pancreatic cancer, including fatty acids, autophagy, macropinocytosis, and deregulated cell-surface glycoproteins, which promotes cancer cell development in an oxygendeficient and nutrient-poor environment.

\section{Altered fatty acid metabolism}

The importance of altered lipid and fatty acid metabolism in cancer has received renewed interest because they are not only components of the membrane matrix and secondary messengers, but also a source of energy (adenosine triphosphate - ATP), and essential for production of cellular membranes, which is especially important for a rapidly growing tumor. Attention has been paid to the ability of cancer cells to rewire lipid metabolism in order to sustain growth, division, and survival [5]. The metabolic hallmark of cancer cells has become lipidomic remodeling, including changes in lipid transport (increase lipid uptake from the environment), storage, de novo lipogenesis, and $\beta$-oxidation (production of ATP) [6]. A high-fat diet that activates oncogenic Kras and COX2 can induce the development of pancreatic cancer in mice. It has also been shown that serum fatty acid synthase (FAS) levels are higher in patients with PDAC and with intraductal papillary mucinous neoplasia (IPMN) of the pancreas, and FAS protein was overexpressed in the ductal epithelium of primary pancreatic adenocarcinomas (86.0\%) and in IPMNs (93.3\%) [7]. Higher expression of FAS is correlated with shorter survival and with a poor response to gemcitabine therapy in pancreatic cancer $[8,9]$. The pharmacological inhibition of this enzyme reduces stemness features and gemcitabine resistance in pancreatic cancer. It has also been demonstrated that up-regulation of fatty acid synthase induced by EGFR/ERK (epidermal growth factor receptor/extracellular signal-regulated kinase) pathway activation promotes tumor growth [10]. In addition, fatty acids' exogenous uptake requires the presence of the transporter CD36, which also influences gemcitabine resistance in PDAC by regulating anti-apoptosis proteins $[11,12]$. Patients with high CD36 expression have lower overall survival, and the use of anti-CD36 strategies could represent a promising therapeutic approach. Recently, a clinical investigation using liquid chromatography tandem mass spectrometry (LC-MS/MS) revealed that differences in lipidomics may be potential biomarkers for early diagnosis of pancreatic cancer [13]. It was found that free fatty acids (FFAs) were significantly decreased in patients with pancreatic cancer. These results also suggest that the damage of the residual lipolysis pathway of aldehyde fatty acid may be related to the development of pancreatic cancer. Previous research suggests that impairment in a lipolytic pathway involving lipases and a unique set of saturated FFAs may play an important role in the development and progression of pan- creatic cancer and provide potential targets for therapeutic intervention [14]. The expression levels of lipase genes are decreased in pancreatic cancer tissues, and the expression of the genes for pancreatic lipase (PNLIP) in tumors is associated with worse patient survival. These studies showed that saturated FFAs can induce TRAIL (TNF-related apoptosis-inducing ligand) expression and apoptosis, and inhibit the proliferation of pancreatic cancer cells.

It is also possible to block the kinases that drive the neoplastic process by applying appropriate nutritional treatment, which is often used in malnourished pancreatic cancer patients requiring extensive surgery. An omega 3-enriched diet led to reduced lesion penetrance via repression of proliferation associated with reduced phosphorylated AKT/ protein kinase B (pAKT), whereas an omega 6-enriched diet accelerated tumor formation [15]. The results of these studies suggested that omega- 3 fatty acids may prevent early pancreatic carcinogenesis. In previous experimental studies, the human pancreatic cancer cell line MIA PaCa-2 incubated with eicosapentaenoic acid (EPA) demonstrated cell cycle arrest and induction of apoptosis [16]. Feeding animals with a diet supplemented with 5\% fish oil, which contains high levels of EPA and docosahexaenoic acid (DHA), also strongly suppresses the growth of MIA PaCA-2 human pancreatic cancer xenografts in athymic nude mice, by inducing oxidative stress and cell death [17]. Furthermore, EPA induces autophagy in these cancer cells and induction of autophagy reduces its ability to induce apoptotic cell death. It is therefore suggested that a combination of EPA with an autophagy inhibitor may be a useful strategy in increasing the therapeutic effectiveness in pancreatic cancer. Another study using omega-3 fatty acid desaturase (Fat-1) in transgenic mice assessed the impact of omega-3 PUFAs on PanINs (pancreatic intraepithelial neoplasia) and their progression to PDAC [18]. Molecular analysis of the pancreas showed significant down-regulation of proliferating cell nuclear antigen, cyclooxygenase-2, 5-lipoxygenase (5-LOX), 5-LOX-activating protein, Bcl-2, and cyclin D1 expression levels in Fat-1-p48Cre/+-LSL-KrasG12D/+ mice compared to $\mathrm{p} 48 \mathrm{Cre} /+-\mathrm{LSLKrasG12D/+} \mathrm{mice.} \mathrm{These} \mathrm{data} \mathrm{highlighted}$ the usefulness of dietary omega-3 FAs for chemoprevention of pancreatic cancer, especially in high-risk individuals. On the other hand, in clinical trials, high omega-3 PUFA, especially marine omega-3 PUFAs, and DHA consumption was associated with a lower risk of pancreatic cancer [19].

\section{Changes of the adaptive metabolic pathways}

In the adaptation mechanism, pancreatic cancer cells also use other sources of nutrients to support their energy requirements. In addition to glycolysis and the pentose phosphate pathway (non-oxidative PPP), a self-digestion process (autophagy) and uptake of external nutrients (macropinocytosis) can also be activated. Lysosome, which is constitutively activated in PDAC, affects both autophagy 
and macropinocytosis [20]. The nutrient salvage pathways fuel tumor growth and generate sugars, amino acids, and fatty acids necessary to reduce stress from cancer cells' malnutrition [21]. Elevated autophagy in PDAC not only plays a key role in removing reactive oxygen species (ROS) and maintaining energy homeostasis but also prevents DNA damage and increases tumor cell proliferation [22]. Inhibition of autophagy may delay the growth of pancreatic tumor xenografts, while mice lacking the autophagy genes (Atg5 or Atg7) were observed to have PanIN low stage premalignant lesions but did not develop highgrade PanIN and PDAC [23, 24]. Pancreatic neoplasms have constitutively activated autophagy (controlled by KRAS and p53), suggesting their sensitivity to inhibition, which leads to an increase in ROS, DNA damage, changes in cellular metabolism, and overgrowth of cytotoxic substances, preventing sustained tumor growth [23]. Hitting this sensitive point by inhibiting autophagy disrupts the adaptive mechanisms of pancreatic cancer and could also potentially alter resistance to other forms of therapy. Deprivation of the main energy sources (glycolysis) in nutrient stress activates AMPK, which activates autophagy, leading to the induction of glycolysis [25-27]. In PDAC, oncogenic KRAS and glutamine deprivation can activate macropinocytosis, but it is not clear how macropinocytosis may affect the glucose metabolism in tumor cells [28, 29]. It has been demonstrated that KRAS promotes autophagy to recycle the metabolites and promotes macropinocytosis to transport extracellular proteins as an amino acid source for the tricarboxylic acid (TCA) cycle to sustain tumor growth [28]. A better understanding of the importance of autophagy in promoting or inhibiting the neoplastic process requires further research. Although enhanced autophagy is detected in PDAC, its elimination contributes to pancreatic cancer initiation [30]. More precisely, the authors of the latest research demonstrated that acute suppression of KRAS (using validated siRNA) further elevated rather than decreased the already high basal levels of autophagy in a panel of human and mouse PDAC cell lines [31]. It also turned out that autophagy was elevated at multiple levels, including increased autophagosome flux, phosphorylation and activation of AMPK and Beclin-1, downregulation of the mTOR pathway, and increased transcription of autophagy-related genes. These studies showed that combinations of pharmacologic inhibitors that concurrently block both ERK (extracellular signal-regulated kinase)/ MAPK (mitogen-activated protein kinase) and autophagic processes that are upregulated in response to ERK inhibition may be effective treatments for PDAC. In another series of studies, it was shown that inhibition of KRAS $\rightarrow$ $\mathrm{RAF} \rightarrow \mathrm{MEK} \rightarrow$ ERK signaling elicits autophagy, a process of cellular recycling that protects pancreatic ductal cancer cells from the cytotoxic effects of KRAS pathway inhibition [32]. This evidence of protective autophagy suggests a novel treatment strategy for RAS-driven cancers (a combination of trametinib plus hydroxychloroquine). As previous research has shown, the ERK/MAPK signaling pathway plays a central role in pancreatic carcinogenesis [33]. This signaling is involved in cell proliferation, differentiation, cell cycle regulation, apoptosis, and in development of various tumor (e.g., breast, lung, colon cancer), and inhibition of the ERK/MAPK signaling pathway can restore tumor cells to a non-transformed state in vitro and suppresses growth of tumors in vivo [34-37]. In patients with advanced pancreatic cancer in a randomized phase II trial, the combination of selumetinib (MEK inhibitor) and MK-2206 (AKT inhibitor) revealed that their efficacy was inferior to FOLFIRINOX and the toxicity was greater [38]. The clinical effect of the dual-targeting strategy involving PI3K/AKT/mTOR and RAS/MEK/ ERK pathways in patients with advanced cancer suggests that dual inhibition of both pathways may potentially exhibit favorable efficacy compared with inhibition of either pathway, but also at the expense of greater toxicity [39]. Unfortunately, in a phase II randomized, double-blind, placebo-controlled trial in patients with previously untreated metastatic adenocarcinoma of the pancreas, oral mitogen/ extracellular signal-related kinase (MEK1/2) inhibitor (trametinib in combination with gemcitabine) had no clinical benefit including overall survival, progression-free survival, overall response rate, and duration of response, and outcomes were independent of KRAS mutations determined by circulating free DNA (cfDNA) [40]. In a phase I/II trial, no clinical benefit was observed also with first-line pimasertib (selective MEK1/2 inhibitor) plus gemcitabine compared with gemcitabine alone in patients with metastatic pancreatic adenocarcinoma [41]. An interesting attempt to inhibit the proliferation of KRAS mutated pancreatic cancer cells is also the inhibition of nutrient uptake by macropinocytosis. Phellodendrine chloride (PC) significantly inhibited the viability of KRAS mutant pancreatic cancer cells (PANC-1 and MiaPaCa-2) in a dose-dependent manner [42]. As shown by these experimental studies, the inhibition of macropinocytosis by PC and the decrease in the intracellular level of glutamine had the effect of limiting PANC-1 cell growth. In PANC-1 cells, inhibition of macropinocytosis led to an enhanced ROS level and induced mitochondrial apoptosis by increased Bax, decreased Bcl-2 expression, and activation of caspase-3, 7, 9, and PARP cleaved. In other studies, it was found that macropinocytosis rendered a subset of pancreatic tumor cells resistant to mTOR inhibition, and concurrent inhibition of mTOR and protein scavenging might be a valuable therapeutic approach [43]. Another interesting discovery was the SDC1 (syndecan-1) protein activated by KRAS on the cell surface that regulates macropinocytosis in pancreatic cancer cells. The determination of this cell surface critical mediator of macropinocytosis may be an important diagnostic and therapeutic element [44]. As cancer cells' metabolic adaptation to alterations in the microenvironment, 
macropinocytosis provides not only a survival mechanism under nutrient-scarce conditions but also the potential for unrestricted tumor growth in an adverse tumor microenvironment.

In metabolic adaptation, deregulated cell-surface proteins drive neoplastic transformations and are envisioned to mediate crosstalk between the tumor and its microenvironment. Numerous studies indicate the important roles of mucins, a family of multifunctional glycoproteins of epithelial cells in the gastrointestinal tract, that not only protect but also participate in increasing carcinogenicity, invasiveness, metastasis, and tumor drug resistance [45-47]. Multiple functional domains present in the mucins (e.g., epidermal growth factor-EGF) interact with cell surface receptors, signaling mediators, and the extracellular matrix (ECM). Aberrant overexpression of the oncoprotein MUC1 is found in $90 \%$ of pancreatic cancer and is associated with a short survival rate [48-50]. MUC1 is an important regulator of metabolic reprogramming (e.g., increased glycolysis), facilitating metabolic adaptation of cancer cells in a hypoxic environment, which helps cancer development. In orthotopic models of pancreatic cancer implantation, MUC1 increases glucose uptake and the expression of genes involved in uptake [51]. The results of these studies indicate that MUC1 acts as a modulator of the hypoxia response of pancreatic cancer cells by regulating the expression and activity of the transcription factor HIF-1. Preclinical studies suggest that decreasing mucin expression in tumors has potential as a novel molecular approach for the treatment of pancreatic cancer [52]. For example, inhibition of MUC1 biosynthesis via threonyl-tRNA synthetase suppresses pancreatic cancer cell migration [53]. Healthy pancreatic tissue may express low levels of mucins such as MUC1, or MUC1 is undetectable in the main pancreatic duct; however, histological studies show that there is neo-expression and upregulation of mucins such as MUC4, MUC5AC, and MUC16 in pancreatic intraepithelial neoplasia (PanIN), intraductal papillary mucinous neoplasms (IPMN), and mucinous cystic neoplasms (MCN) [48, 49, 54, 55]. Histological studies revealed a multifold increase in MUC1 and MUC4 expression as early as the PanIN lesion stage (in $85 \%$ of cases, PDAC arises from PanIN), which further increases during pancreatic cancer progression. The highest expression of MUC1, MUC4, and MUC5AC was found in PanIN III and pancreatic cancer [47, 55-58]. In immunocytochemistry, MUC4 and MUC16 have high specificity in distinguishing PDAC patients from healthy controls, and the latest research showed that MUC4 levels in the cystic fluid of IPMN patients could accurately discriminate high- from low-risk cystic neoplasms with high sensitivity $[59,60]$. In pancreatic cancer, MUC1 binds to $\beta$-catenin and EGFR to activate cell proliferation via the $\mathrm{Wnt} / \beta$-catenin or MAPK pathway [61, 62], and up-regulation of MUC16 stimulates mTOR and c-MYC to reprogram pancreatic cancer metabolism, enhancing glycolysis and cell proliferation, and can also activate the
AKT and MAPK pathways to promote metastasis [63, 64]. In pancreatic cancer cells, MUC13 expression leads to the activation of nuclear translocation of NF- $\mathrm{BB}$ p65 and phosphorylation of $\mathrm{I} \kappa \mathrm{B}$, which in turn upregulates the expression of important proteins (Glut-1, c-Myc, and Bcl-2) that are involved in glucose metabolism [65]. These results delineate how MUC13 rewires aberrant glucose metabolism to enhance the aggressiveness of pancreatic cancer and reveal a novel mechanism to develop newer therapeutic strategies.

Mucins are also a target for the development of new therapies as well as to enhance the potency of cytotoxic drugs $[62,66,67]$. Mucin-based therapy has been applied for pancreatic cancer strategies including vaccines, antibodies, gene therapy, and mucolytic agents. For example, in clinical application, a synthetic MUC1 peptide was used as a vaccine to strengthen the tumor-associated antigen presentation of dendritic cells and to activate cytotoxic $\mathrm{T}$ cells in resected and locally advanced pancreatic cancer [68]. Pancreatic cancer immunotherapy with MUC1-based vaccines reduced the tumor burden by inducing a cellular and humoral response [69]. The combination of MUC1 antibodies and radioisotopes is an interesting concept, but the side effects of radiotherapy such as neutropenia and thrombocytopenia as well as the high cost should be considered [70, 71]. Down-regulation of MUC1 expression by RNA interference reduces the growth rate and metastatic phenotype of human pancreatic cancer cells [72]. These results support the hypothesis that MUC1 contributes significantly to growth and metastasis, and that down-regulation of MUC1 protein expression decreases the metastatic potential of pancreatic adenocarcinoma. In an orthotopic mouse model, MUC4 interference by siRNA also inhibited pancreatic tumor cell proliferation [72]. The presence of MUC4 on the cell surface was shown to interfere with the tumor cell-extracellular matrix interactions, in part by inhibiting the integrin-mediated cell adhesion. These studies provide experimental evidence that supports the functional significance of MUC4 in pancreatic cancer progression and indicates a novel role for MUC4 in cancer cell signaling. In another study, immunohistochemical staining demonstrated that high MUC20 expression correlated with poor progression-free survival and a high local recurrence rate of PDAC patients, and MUC20 knockdown with siRNA suppresses the malignant phenotypes of PDAC cells at least partially through inhibition of hepatocyte growth factor (HGF)/MET signaling, a critical pathway for communication between stroma and cancer cells, and that MUC20 could act as a potential therapeutic target [73]. Despite the encouraging results of experimental studies, $M U C$ gene silencing therapy has not been confirmed in clinical trials.

\section{Conclusions}

Hypoxia, impaired perfusion, and the limited access of PDAC cells to nutrients stimulates development of ad- 
aptations (e.g., autophagy/recycling, macropinocytosis/ extracellular scavenging) to meet the basic energy requirements and the needs of unconstrained proliferation. It was found that blocking of selected metabolic pathways related to the local adaptive metabolic activity of pancreatic cancer cells, improving nutrient acquisition and metabolic crosstalk within the microenvironment to sustain proliferation, may inhibit cancer development, increase cancer cell death, and increase sensitivity to other forms of treatment (e.g., chemotherapy). Most of these studies have been performed on different pancreatic cancer cell lines, under varying experimental conditions, significantly different from the conditions of the gradual development of neoplastic lesions in pancreatic cancer patients, making it difficult to compare and transfer the results of these studies to clinical practice. In addition, analyses are confounded by the heterogeneous populations of cells found in the tumor microenvironment. The changes in the metabolic profile can also be caused by non-cancerous cells strongly supporting tumor growth. Thus, the assessment of the contribution of metabolic disorders to the development of pancreatic cancer should also take into account other factors explaining the roles of different cellular compartments in cancer development (stroma, immune cells), which may alter metabolism in vivo. Without a better understanding of the mechanisms of the tumor metabolism adjustment to the changing conditions that inhibit its development, it will be impossible to develop a more effective therapy. Unfortunately, in clinical trials conducted in patients with advanced pancreatic cancer, there was no advantage of treatment including various combinations of metabolic signaling pathway inhibitors, over the current standard treatment, but studies are still ongoing.

\section{The authors declare no conflict of interest.}

\section{References}

1. Siegel RL, Miller KD, Jemal A (2020): Cancer statistics 2020. CA Cancer J Clin 70: 7-30.

2. Ducreux M, Seufferlein T, Laethem JL, et al. (2019): Systemic treatment of pancreatic cancer revisited. Semin Oncol 46: $28-38$.

3. Reni M, Riess H, O'Reilly EM, et al. (2020): Phase III APACT trial of adjuvant $n a b$-paclitaxel plus gemcitabine $(n a b-\mathrm{P}+\mathrm{Gem})$ versus gemcitabine $(\mathrm{Gem})$ alone for patients with resected pancreatic cancer (PC): Outcomes by geographic region. J Clin Oncol 38 (15 suppl.): 4515-4515.

4. Biancur DE, Kimmelman AC (2018): The plasticity of pancreatic cancer metabolism in tumor progression and therapeutic resistance. Biochim Biophys Acta Rev Cancer 1870: 67-75.

5. Beloribi-Djefaflia S, Vasseur S, Guillaumond F (2016): Lipid metabolic reprogramming in cancer cells. Oncogenesis 5: e189.

6. Rohrig F, Schulze A (2016): The multifaceted roles of fatty acid synthesis in cancer. Nat Rev Cancer 16: 732-749.
7. Walter K, Hong SM, Nyhan S, et al. (2009): Serum fatty acid synthase as a marker of pancreatic neoplasia. Cancer Epidemiol Biomarkers Prev 18: 2380-2385.

8. Tadros S, Shukla SK, King RJ, et al. (2017): De novo lipid synthesis facilitates gemcitabine resistance through endoplasmic reticulum stress in pancreatic cancer. Cancer Res 77: 5503-5517.

9. Yang Y, Liu H, Li Z, et al. (2011): Role of fatty acid synthase in gemcitabine and radiation resistance of pancreatic cancers. Int J Biochem Mol Biol 2: 89-98.

10. Bian Y, Yu Y, Wang S, Li L (2015): Up-regulation of fatty acid synthase induced by EGFR/ERK activation promotes tumor growth in pancreatic cancer. Biochem Biophys Res Commun 463: 612-617.

11. Kubo M, Gotoh K, Eguchi H, et al. (2019): Impact of CD36 on chemoresistance in pancreatic ductal adenocarcinoma. Ann Surg Oncol 27: 610-619.

12. Kubo M, Eguchi H (2019): ASO author reflections: regulation of chemoresistance in pancreatic ductal adenocarcinoma by scavenger receptor CD36. Ann Surg Oncol 27: 620-621.

13. Zhou D, Mu D, Cheng M, et al. (2020): Differences in lipidomics may be potential biomarkers for early diagnosis of pancreatic cancer. Acta Cir Bras 35: e202000508.

14. Zhang G, He P, Tan H, et al. (2013): Integration of metabolomics and transcriptomics revealed a fatty acid network exerting growth inhibitory effects in human pancreatic cancer. Clin Cancer Res 19: 4983-4993.

15. Ding Y, Mullapudi B, Torres C, et al. (2018): Omega-3 fatty acids prevent early pancreatic carcinogenesis via repression of the AKT pathway. Nutrients 10: 1289.

16. Lai PBS, Ross JA, Fearon KCH, et al. (1996): Cell cycle arrest and induction of apoptosis in pancreatic cancer cells exposed to eicosapentaenoic acid in vitro. $\mathrm{Br} \mathrm{J}$ Cancer 74 : 1375-1383.

17. Fukui M, Kang KS, et al. (2013): EPA, an omega-3 fatty acid, induces apoptosis in human pancreatic cancer cells: role of ROS accumulation, caspase-8 activation, and autophagy induction. J Cell Biochem 114: 192-203.

18. Mohammed A, Janakiram NB, Brewer M, et al. (2012): Endogenous $n-3$ polyunsaturated fatty acids delay progression of pancreatic ductal adenocarcinoma in Fat-1-p48(Cre/+)-LSL$\mathrm{Kras}(\mathrm{G} 12 \mathrm{D} /+)$ mice. Neoplasia 14: 1249-1259.

19. Hidaka A, Shimazu T, Sawada N, et al. (2015): Fish, n-3 PUFA consumption, and pancreatic cancer risk in Japanese: a large, population-based, prospective cohort study. Am J Clin Nutr 102: 1490-1497.

20. Perera RM, Stoykova S, Nicolay BN, et al. (2015): Transcriptional control of autophagy-lysosome function drives pancreatic cancer metabolism. Nature 524: 361-365.

21. Yan L, Raj P, Yao W, Ying H (2019): Glucose metabolism in pancreatic cancer. Cancers 11: 1460.

22. Yang S, Kimmelman AC (2011): A critical role for autophagy in pancreatic cancer. Autophagy 7: 912-913.

23. Yang S, Wang X, Contino G, et al. (2011): Pancreatic cancers require autophagy for tumor growth. Genes Dev 25: 717-729.

24. Rosenfeldt MT, O'Prey J, Morton JP, et al. (2013): P53 status determines the role of autophagy in pancreatic tumour development. Nature 504: 296-300.

25. Kim J, Kim YC, Fang C, et al. (2013): Differential regulation of distinct Vps34 complexes by AMPK in nutrient stress and autophagy. Cell 152: 290-303. 
26. Williams T, Forsberg LJ, Viollet B, Brenman JE (2009): Basal autophagy induction without AMP-activated protein kinase under low glucose conditions. Autophagy 5: 1155-1165.

27. Lock R, Roy S, Kenific CM, et al. (2011): Autophagy facilitates glycolysis during Ras-mediated oncogenic transformation. Mol Biol Cell 22: 165-178.

28. Commisso C, Davidson SM, Soydaner-Azeloglu RG, et al. (2013): Macropinocytosis of protein is an amino acid supply route in Ras-transformed cells. Nature 497: 633-637.

29. Lee SW, Zhang Y, Jung M, et al. (2019): EGFR-pak signaling selectively regulates glutamine deprivation-induced macropinocytosis. Dev Cell 50: 381-392.

30. New M, Van Acker T, Long JS, et al. (2017): Molecular pathways controlling autophagy in pancreatic cancer. Front Oncol 7: 28.

31. Bryant KL, Stalnecker CA, Zeitouni D, et al. (2019). Combination of ERK and autophagy inhibition as a treatment approach for pancreatic cancer. Nat Med 25: 628-640.

32. Kinsey CG, Camolotto SA, Boespflug AM, et al. (2019): Protective autophagy elicited by RAF $\rightarrow$ MEK $\rightarrow$ ERK inhibition suggests a treatment strategy for RAS-driven cancers. Nat Med 25: 620-627.

33. Collisson EA, Trejo C, Silva J, et al. (2012): A central role for $\mathrm{RAF} \rightarrow \mathrm{MEK} \rightarrow \mathrm{ERK}$ signaling in the genesis of pancreatic ductal adenocarcinoma. Cancer Discov 2: 685-693.

34. Rao A, Herr DR (2017): G protein-coupled receptor GPR19 regulates E-cadherin expression and invasion of breast cancer cells. Biochim Biophys Acta Mol Cell Res 1864: 1318-1327.

35. Tang Q, Wu J, Zheng F, et al. (2017): Emodin increases expression of insulin-like growth factor binding protein 1 through activation of MEK/ERK/AMPK $\alpha$ and interaction of PPAR $\gamma$ and Sp1 in lung cancer. Cell Physiol Biochem 41: 339-357.

36. Rubinfeld H, Seger R (2005): The ERK cascade: A prototype of MAPK signaling. Mol Biotechnol 31: 151-174.

37. Sebolt-Leopold JS, Dudley DT, Herrera R, et al. (1999): Blockade of the MAP kinase pathway suppresses growth of colon tumors in vivo. Nat Med 5: 810-816.

38. Chung V, McDonough S, Philip PA, et al. (2017): Effect of selumetinib and MK-2206 vs oxaliplatin and fluorouracil in patients with metastatic pancreatic cancer after prior therapy: SWOG S1115 study randomized clinical trial. JAMA Oncol 3: 516-522.

39. Shimizu T, Tolcher AW, Papadopoulos KP, el al. (2012): The clinical effect of the dual-targeting strategy involving $\mathrm{PI} 3 \mathrm{~K} / \mathrm{AKT} / \mathrm{mTOR}$ and RAS/MEK/ERK pathways in patients with advanced cancer. Clin Cancer Res 18: 2316-2325.

40. Infante JR, Somer BG, Park JO, et al. (2014): A randomised, double-blind, placebo-controlled trial of trametinib, an oral MEK inhibitor, in combination with gemcitabine for patients with untreated metastatic adenocarcinoma of the pancreas. Eur J Cancer 50: 2072-2081.

41. Cutsem EV, Hidalgo M, Canon JL, et al. (2018): Phase I/II trial of pimasertib plus gemcitabine in patients with metastatic pancreatic cancer. Int J Cancer 143: 2053-2064.

42. Thu PM, Zheng ZG, Zhou YP, et al. (2019): Phellodendrine chloride suppresses proliferation of KRAS mutated pancreatic cancer cells through inhibition of nutrients uptake via macropinocytosis. Eur J Pharmacol 850: 23-34.

43. Michalopoulou E, Auciello FR, Bulusu V, et al. (2020): Macropinocytosis renders a subset of pancreatic tumor cells resistant to mTOR inhibition. Cell Rep 30: 2729-2742.
44. Yao W, Rose JL, Wang W, et al. (2019): Syndecan1 is a critical mediator of macropinocytosis in pancreatic cancer. Nature 568: 410-414

45. Bafna S, Kaur S, Batra SK (210): Membrane-bound mucins: the mechanistic basis for alterations in the growth and survival of cancer cells. Oncogen 29: 2893-2904.

46. Banerjee S, Mujumdar N, Dudeja V, et al. (2012): MUC1c regulates cell survival in pancreatic cancer by preventing lysosomal permeabilization. PLoS One 7: e43020.

47. Wang S, You L, Dai M, Zhao Y (2020): Mucins in pancreatic cancer: A well-established but promising family for diagnosis, prognosis and therapy. J Cell Mol Med 24: 10279-10289.

48. Torres MP, Chakraborty S, Souchek J, Batra SK (2012): Mucin-based targeted pancreatic cancer therapy. Curr Pharm Des 18: 2472-2481.

49. Kaur S, Kumar S, Momi N, Sasson AR, et al. (2013): Mucins in pancreatic cancer and its microenvironment. Nat Rev Gastroenterol Hepatol 10: 607-620.

50. Nath S, Mukherjee P (2014): MUC1: a multifaceted oncoprotein with a key role in cancer progression. Trends $\mathrm{Mol} \mathrm{Med}$ 20: 332-342.

51. Chaika NV, Gebregiworgisb T, Lewallena ME, et al. (2012): MUC1 mucin stabilizes and activates hypoxia-inducible factor 1 alpha to regulate metabolism in pancreatic cancer. PNAS 109: 13787-13792.

52. Tsutsumida H, Swanson BJ, Singh PK, et al. (2006): RNA interference suppression of MUC1 reduces the growth rate and metastatic phenotype of human pancreatic cancer cells. Clin Cancer Res 12: 2976-2987.

53. Jeong SJ, Kim JH, Lim BJ, et al. (2018): Inhibition of MUC1 biosynthesis via threonyl-tRNA synthetase suppresses pancreatic cancer cell migration. Exp Mol Med 50: e424.

54. Moniaux N, Chaturvedi P, Varshney GC, et al. (2007): Human MUC4 mucin induces ultra-structural changes and tumorigenicity in pancreatic cancer cells. Br J Cancer 97: 345357.

55. Nagata K, Horinouchi M, Saitou M, et al. (2007): Mucin expression profile in pancreatic cancer and the precursor lesions. J Hepatobiliary Pancreat Surg 14: 243-254.

56. Suh H, Pillai K, Morris DL, et al. (2017): Mucins in pancreatic cancer: biological role, implications in carcinogenesis and applications in diagnosis and therapy. Am J Cancer Res 7: 1372-1383

57. Yonezawa S, Higashi M, Yamada N, et al. (2010): Significance of mucin expression in pancreatobiliary neoplasms. J Hepatobiliary Pancreat Sci 17: 108-124.

58. Yonezawa S, Taira M, Osako M, et al. (1998): MUC-1 mucin expression in invasive areas of intraductal papillary mucinous tumors of the pancreas. Pathol Int 48: 319-322.

59. Horn A, Chakraborty S, Dey P, et al. (2013):Immunocytochemistry for MUC4 and MUC16 is a useful adjunct in the diagnosis of pancreatic adenocarcinoma on fine-needle aspiration cytology. Arch Pathol Lab Med 137: 546-551.

60. Maker AV, Hu V, Kadkol SS, et al. (2019): Cyst fluid biosignature to predict intraductal papillary mucinous neoplasms of the pancreas with high malignant potential. J Am Coll Surg 228: 721-729.

61. Nath S, Roy LD, Grover P, et al. (2015): Mucin 1 regulates cox-2 gene in pancreatic cancer. Pancreas 44: 909-917.

62. Trehoux S, Duchene B, Jonckheere N, Van Seuningen I (2015): The MUC1 oncomucin regulates pancreatic cancer cell biological properties and chemoresistance. Implication of 
p42-44 MAPK, Akt, Bcl-2 and MMP13 pathways. Biochem Biophys Res Commun 456: 757-762.

63. Muniyan S, Haridas D, Chugh S, et al. (2016): MUC16 contributes to the metastasis of pancreatic ductal adenocarcinoma through focal adhesion mediated signaling mechanism. Genes Cancer 7: 110-124.

64. Shukla SK, Gunda V, Abrego J, et al. (2015): MUC16-mediated activation of mTOR and c-Myc reprograms pancreatic cancer metabolism. Oncotarget 6: 19118-19131.

65. Kumari S, Khan S, Gupta SC, et al. (2018): MUC13 contributes to rewiring of glucose metabolism in pancreatic cancer. Oncogenesis 7: 19.

66. Nath S, Daneshvar K, Roy LD, et al. (2013): MUC1 induces drug resistance in pancreatic cancer cells via upregulation of multidrug resistance genes. Oncogenesis 2: e51.

67. Skrypek N, Duchęne B, Hebbar M, et al. (2013): The MUC4 mucin mediates gemcitabine resistance of human pancreatic cancer cells via the concentrative nucleoside transporter family. Oncogene 32: 1714-1723.

68. Ramanathan RK, Lee KM, McKolanis J, et al. (2005): Phase I study of a MUC1 vaccine composed of different doses of MUC1 peptide with SB-AS2 adjuvant in resected and locally advanced pancreatic cancer. Cancer Immunol Immunother 54: 254-264.

69. Li M, Wang Z, Yan B, et al. (2019): Design of a MUC1-based tricomponent vaccine adjuvanted with FSL-1 for cancer immunotherapy. Medchemcomm 10: 2073-2077.

70. Cardillo TM, Ying Z, Gold DV (2001): Therapeutic advantage of (90)yttrium- versus (131)iodine-labeled PAM4 antibody in experimental pancreatic cancer. Clin Cancer Res 7: 3186-3192.

71. Han S, Jin G, Wang L, et al. (2014): The role of PAM4 in the management of pancreatic cancer: diagnosis, radioimmunodetection, and radioimmunotherapy. J Immunol Res 2014: 268479.

72. Chaturvedi P, Singh AP, Moniaux N, et al. (2007): MUC4 mucin potentiates pancreatic tumor cell proliferation, survival, and invasive properties and interferes with its interaction to extracellular matrix proteins. Mol Cancer Res 5: 309-320.

73. Chen ST, Kuo TC, Liao YY, et al. (2018): Silencing of MUC20 suppresses the malignant character of pancreatic ductal adenocarcinoma cells through inhibition of the HGF/ MET pathway. Oncogene 37: 6041-6053. 